\title{
ON-ORBIT SOLAR DYNAMICS OBSERVATORY (SDO) STAR TRACKER WARM PIXEL ANALYSIS
}

\author{
Denis Felikson, ${ }^{(1)}$ Matthew Ekinci, ${ }^{(2)}$ Joseph A. Hashmall, ${ }^{(3)}$ and Melissa Vess ${ }^{(4)}$ \\ (1) a.i. solutions, Inc., 10001 Derekwood Lane, Lanham, MD 20706, USA, denis.felikson@ai-solutions.com \\ (2) NASA / Goddard Space Flight Center, Code 444, Greenbelt, MD 20771, USA, f.matthew.ekinci@nasa.gov \\ (3) a.i. solutions, Inc., 10001 Derekwood Lane, Lanham, MD 20706, USA, joseph.hashmall@ai-solutions.com \\ ${ }^{(4)}$ NASA / Goddard Space Flight Center, Code 591, Greenbelt, MD 20771, USA, melissa.f.vess@nasa.gov
}

\begin{abstract}
This paper describes the process of identification and analysis of warm pixels in two autonomous star trackers on the Solar Dynamics Observatory (SDO) mission. A brief description of the mission orbit and attitude regimes is discussed and pertinent star tracker hardware specifications are given. Warm pixels are defined and the Quality Index parameter is introduced, which can be explained qualitatively as a manifestation of a possible warm pixel event. A description of the algorithm used to identify warm pixel candidates is given. Finally, analysis of dumps of on-orbit star tracker charge coupled devices (CCD) images is presented and an operational plan going forward is discussed.
\end{abstract}

SDO, launched on February 11, 2010, is operated from the NASA Goddard Space Flight Center (GSFC). SDO is in a geosynchronous orbit with a $28.5^{\circ}$ inclination. The nominal mission attitude points the spacecraft $\mathrm{X}$-axis at the Sun, with the spacecraft Z-axis roughly aligned with the Solar North Pole. The spacecraft Y-axis completes the triad. In attitude, SDO moves approximately $0.04^{\circ}$ per hour, mostly about the spacecraft $Z$-axis.

The SDO star trackers, manufactured by Galileo Avionica, project the images of stars in their $16.4^{\circ} \times 16.4^{\circ}$ fields-of-view onto CCD detectors consisting of $512 \times 512$ pixels. The trackers autonomously identify the star patterns and provide an attitude estimate. Each unit is able to track up to 9 stars. Additionally, each tracker calculates a parameter called the Quality Index, which is a measure of the quality of the attitude solution. Each pixel in the CCD measures the intensity of light and a warm pixel is defined as having a measurement consistently and significantly higher than the mean background intensity level. A warm pixel should also have lower intensity than a pixel containing a star image and will not move across the field of view as the attitude changes (as would a dim star image). It should be noted that the maximum error introduced in the star tracker attitude solution during suspected warm pixel corruptions is within the specified $3 \sigma$ attitude error budget requirement of $[35,70,70]$ arcseconds. Thus, the star trackers provided attitude accuracy within the specification for SDO.

The star tracker images are intentionally defocused so each star image is detected in more than one CCD pixel. The position of each star is calculated as an intensity-weighted average of the illuminated pixels. The exact method of finding the positions is proprietary to the tracker manufacturer. When a warm pixel happens to be in the vicinity of a star, it can corrupt the calculation of the position of that particular star, thereby corrupting the estimate of the attitude. 
Figure 1 below shows the Quality Index from one of the star trackers during a period of time when a warm pixel is suspected to have corrupted the attitude solution. The Quality Index has a distinct "inverted-U" shape which was observed during several suspected warm pixel events. This paper explains the causal connection between this pattern in the Quality Index due to a warm pixel corruption of the attitude solution.

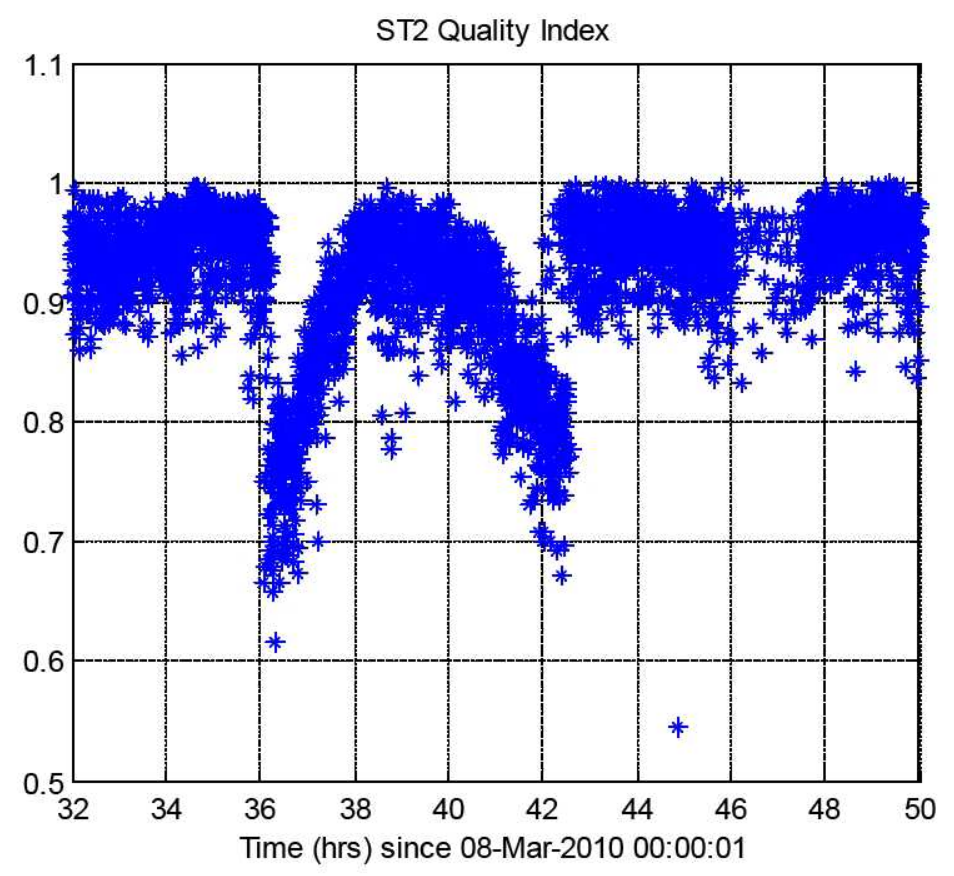

Figure 1. Star Tracker 2 Quality Index from 09-Mar-2010 08:00 to 10-Mar-2010 02:00 (UTC)

The algorithm implemented to detect possible warm pixels uses CCD dumps of the pixel intensity for a star tracker in a comparison with previous dumps. The procedure is:

1. The intensities of each pixel are compared with a threshold intensity and those above that threshold are considered to contain star images.

2. The pixels containing star images and those surrounding the central star image pixel are eliminated from further analysis.

3. Pixels that are not proximate to star images are considered background and the mean and standard deviations of the background is calculated.

4. Any pixels that are above a statistical threshold (n $\sigma)$ over the mean background are considered candidates for being warm pixels.

5. If a pixel is a candidate for being warm in two successive dumps, it is recorded as a possible warm pixel.

6. As additional dumps are accumulated, a record of the number of times any pixel was designated a possible warm pixel is maintained. 
The repeatability of designation of a pixel as a possible warm pixel provides the analyst a basis for judgment of which pixels are actually and consistently warm.

When this anomaly was first recognized, the entire CCD image from Star Tracker 2 was dumped 3 times on different days. Initial analysis of these 3 CCD images, using the algorithm described above, revealed pixels which had an intensity of greater than $6 \sigma$ over the mean background intensity in all 3 CCD images. These pixels were the initial warm pixel suspects because they met the criteria for measuring intensity significantly higher than the background intensity consistently in 3 different CCD dumps at somewhat different attitudes. Additionally, this paper presents interesting aspects of the background intensity distributions from the 3 CCD images combined.

Since launch, a total of $8 \mathrm{CCD}$ dumps per tracker have been performed. The first series of dumps was performed in late March of 2010 (roughly 5 weeks after launch), with subsequent dumps being performed once per month per tracker beginning in July of 2010. SDO's Star Tracker 1 CCD has been relatively stable over this span, with the software having found only 8 warm pixels in total and having seen no more than 1 new warm pixel in 6 of the 7 comparison dumps. Of the 8 warm pixel candidates, only 4 have been seen more than once, and only 2 have been seen in the majority of the dumps. Results for Star Tracker 2 have proven much more erratic, with 3 or more new possible warm pixels found in 6 of the 7 comparison dumps and 41 total warm pixel candidates found. However, of these 41 pixels, 28 have been seen in only 1 or 2 of the dumps, with 4 pixels having been seen in 5 or more dumps.

It is possible that the star threshold or the sigma multiplier or both for Star Tracker 2 may need to be modified to provide more consistent identification of warm pixels. Further analysis and operational plans for managing warm pixels on SDO star trackers are discussed in this paper. Beyond monitoring the evolution of potential warm pixels, mitigation plans include potentially augmenting the star tracker "hot-pixel" table with suspected warm pixels. The "hot pixel" table is used by the star tracker to exclude specific pixels from use in determining the star tracker solution. In general, the star tracker will only update the hot pixel table if it determines that a given pixel has an energy level that is well beyond the background. The table can be modified via command from the ground.

The Lunar Reconnaissance Orbiter (LRO), also operated from GSFC and launched in June 2009, uses two of the same model star trackers as the primary attitude knowledge sensors. The Quality Indices from the LRO star trackers have exhibited behavior that could be interpreted as arising from a similar warm pixel effect but, because the attitude of LRO changes rapidly, the effect is more difficult to characterize. This paper discusses the differences in orbit and attitude regimes between the two spacecraft. Because of these differences, the possible warm pixel effect on LRO's attitude accuracy is short-lived compared to SDO.

Although the warm pixel anomaly was observed in these particular star trackers, it may be a characteristic of all CCD star trackers and similar devices. The attitude accuracy of SDO was minimally affected by this anomaly and remained within attitude accuracy requirements. The SDO flight software already includes logic to reject star tracker solutions whose quality indices are below a specified threshold. However, missions with tighter knowledge tolerances may exceed attitude accuracy limits due to a warm pixel effect and may need to mitigate the issue by 
using similar operational procedures as used for SDO or by including logic in flight software to handle warm pixels. 